\title{
High-precision amplifiers for strain gauge based transducers - first time realized in compact size
}

\author{
André Schäfer \\ Hottinger Balwin Messtechnik GmbH (HBM), Im Tiefen See 45, 64293 Darmstadt, Germany
}

\section{ABSTRACT}

In cases where accuracy matters, and in metrology of mechanical quantities such as force, torque or pressure this is definitely the case, are widely realized by transducers, which are based on strain gauges. To exploit the potential of the widely used high-precision static measurements the demands on precision amplifiers steadily grow. This paper gives exact details to further progress of the precision amplifiers used and how compact they can be realized nowadays.

Keywords: high-precision amplifiers; strain gauges; traceability; force; torque; pressure

Citation: André Schäfer, High-precision amplifiers for strain gauge based transducers -first time realized in compact size, Acta IMEKO, vol. 6, no. 4, article 5, December 2017, identifier: IMEKO-ACTA-06 (2017)-04-05

Editor: Alexandru Salceanu, Technical University of Iasi, Romania

Received May 5, 2017; In final form July 4, 2017; Published December 2017

Copyright: @ 2017 IMEKO. This is an open-access article distributed under the terms of the Creative Commons Attribution 3.0 License, which permits unrestricted use, distribution, and reproduction in any medium, provided the original author and source are credited

Corresponding author: Dr.-Ing. André Schäfer, e-mail: andre.schaefer@hbm.com

\section{INTRODUCTION}

Strain gauge precision transducers are always based on a Wheatstone bridge. By the help of strain gauges (S.G.) arranged in a Wheatstone bridge, mechanical quantities such as force, pressure, torque generating strain can be converted into a variation of resistance and later to a voltage (Figure 1).

This Wheatstone bridge is necessary, as the pure change of resistance is far too small. However the unbalance of the Wheatstone bridge, comparable to a double voltage divider, allows an output signal of nominal $2 \mathrm{mV} / \mathrm{V}$ excitation voltage $\mathrm{U}_{\mathrm{B}}$.

The excellent read-out of the signal benefits from two main principles used in the instrument, which should be briefly explained here as they are the basis for the next sections.

In the elastic deformation range of materials the methods of

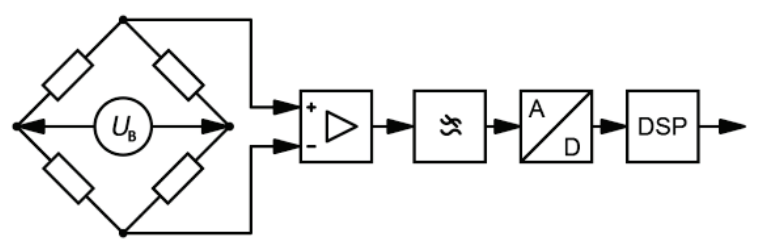

Figure 1. Schematic view of the strain gauge amplifier. calculating the material stresses from the measured strains are based on Hooke's law. In its simplest form for uniaxial stress state Hooke's law is [1], [2]:

$\sigma=E \cdot \varepsilon$

with:

$\sigma=$ material stress

$E=$ modulus of elasticity of the material

$\varepsilon=$ strain.

The variation of resistance is defined by

$\Delta R / R_{0}=k \cdot \varepsilon$

with:

$\Delta R=$ change of resistance

$\mathrm{R}_{0}=$ basic resistance

$\varepsilon=$ strain

$k=$ gauge factor.

This bridge can be extended by useful outer circuitry to "trim" the transducer. For instance zero-point, linearity as well as the temperature compensation of zero point ( $\left.\mathrm{TC}_{\text {zero }}\right)$ and span ( $\left.\mathrm{TC}_{\text {span }}\right)$ [3], [4] can be matched (Figure 2).

The bridge can be supplied by DC or better AC with a frequency $\left(f_{c}\right)$ as "carrier". Now we have to distinguish between the frequency of the carrier and those of the measuring signal. The mechanical quantity changes with the signal frequency $\left(f_{\mathrm{m}}\right)$. The transducer is supplied with an alternating excitation voltage 


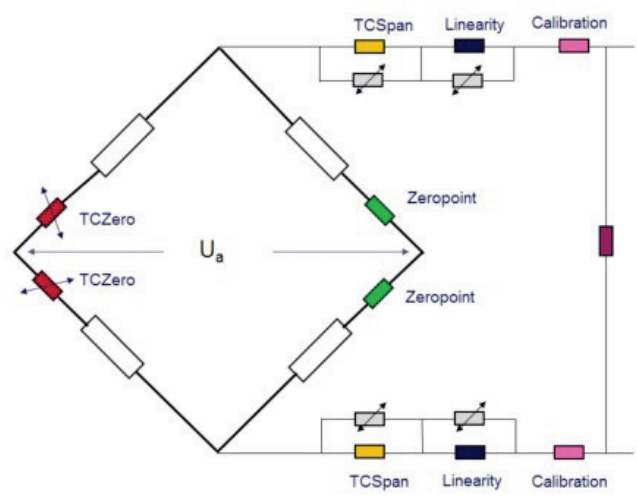

Figure 2. Internal wiring of a precision force transducer.

and works as a modulator. In this way, an interfering signal (AC or DC) can be separated from the measurement signal of the same frequency by the carrier frequency method.

Precision measurements are carried out preferably at $f_{\mathrm{c}}$ equal $225 \mathrm{~Hz}$ and voltage conditions of $2 \mathrm{mV} / \mathrm{V}$. The frequency of $225 \mathrm{~Hz}$ has been chosen by many National Metrology Institutes (NMI's) around the world, as it is no multiple of the disturbing $50 \mathrm{~Hz}$ or $60 \mathrm{~Hz}$ power supply understanding that it is only allowing a signal bandwidth of approx. $50 \mathrm{~Hz}$, so really static measurement, but on the other hand with very high resolution. This very high resolution is first priority, as it is the basis for the reachable accuracy.

At the same time it allows a bandwidth suitable for measurement processing of static and quasi-static signals.

Another reason is that it keeps the influence of noise low. In Figure 3 the spectra of the used carrier frequency principle can be seen. In the graph on the left side the dotted line 6 describes the noise spectrum. With the existing $1 / \mathrm{f}$ noise function (see No. 6 in Figure 3) the choice of $225 \mathrm{~Hz}$ ensures, that one is definitely in the low noise, so above the area of significant noise, which occurs close to $0 \mathrm{~Hz}$.

\section{BASICS AND RELEVANCE OF THE TOPIC}

Traceability of mechanical quantities is relevant to many fields of industry. To name an example, torque measurement is essential for power as well as efficiency measurement and thus to the degree of carbon monoxide pollution and nitrogen oxides in the air.

The same is true for force transducers, which in combination with actuators, are components for all kind of test

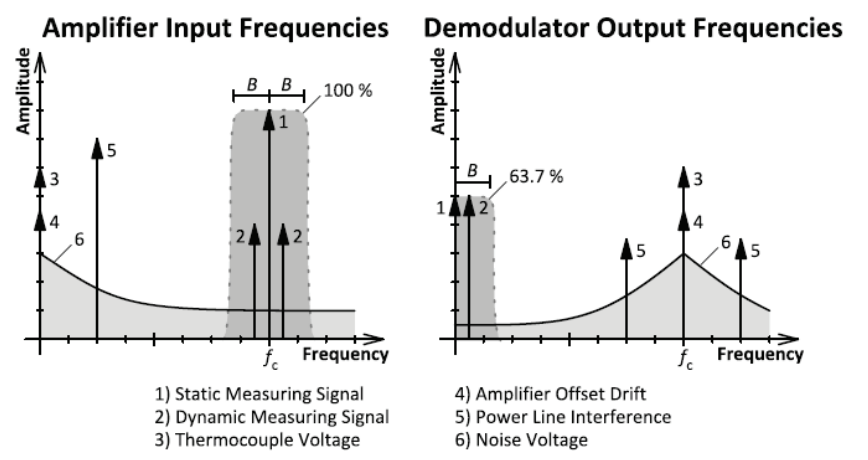

Figure 3. Amplifier input and demodulator frequencies by using carrier frequency principle. stands [5].

Another example is the use of pressure transducers as altimeter in aircrafts, so in an in-flight application. National flight authorities often carry out inter-comparisons using HBM calibration equipment such as the DMP precision amplifier series.

In calculating uncertainty of the whole measuring chain using HBM precision amplifiers you will notice that the overall uncertainty of the measuring chain is often required to be very close or at least in the range of the measurement uncertainty of the transducer itself.

To safeguard critical applications the quality and performance of any kind of calibration equipment is getting more and more important [6], [7], [8].

Due to its worldwide need metrological applications are the most important application fields of precision measurement. In metrological applications one uses the term "calibration pyramid", expressing that the precision of the measurement should correspond to the level of calibration needed.

HBM has taken part in numerous EURAMET driven metrology projects, in various research fields such as measurement uncertainties for measurements of very large force, torque and pressure values, so $\mathrm{MN}$ forces, $\mathrm{MNm}$ torque values as well as pressures in the GPa range.

Another subject we deal with is the challenge of dynamic calibration and here again mainly on mechanical quantities, all to be understood as an especially pioneering field of basic research in the field of metrology of mechanical quantities [9], [10].

\section{NEW COMPACT SIZE MODULES}

This chapter will explain how it was possible to develop such a compact and still very accurate, new type of precision amplifier for strain gauge based transducers.

Despite we have been able to build amplifiers "on the physical limit" down to an accuracy of 5 ppm as early as 1981 (birth date of DMP series), for a better traceability of mechanical quantities in industrial applications; there is also the requirement of building up measuring chains of a total uncertainty of "only" $100 \mathrm{ppm}$.

This total uncertainty should not be evenly distributed to transducer and amplifier, but the requirement to the amplifier is significantly higher, so we put $25 \mathrm{ppm}$ as our goal.

This idea was set by HBM already in the early 1980ies, and created some series of models with "...38" in their name, such as the digital comparators DK 38 introduced in 1982, DK 39 introduced in 1985, MGCplusML38 introduced in 1995 and MGCplusML38B introduced in 2005.

This way HBM was creating its own class of legendary precision amplifiers. During the years the seemingly constant demand for an accuracy of $25 \mathrm{ppm}$ has actually become an increasingly higher requirement, as the requirements of the electromagnetic compatibility over all the time steadily increased.

Of course miniaturization and compact size per channel is a requirement of many calibration applications. In most calibration applications two channels have to be compared ("actio = reactio"), e.g. a reference force transducer and a specimen pressed on each other in a deadweight or hydraulic force calibration machine.

Thus a new amplifier module should feature two independent (simultaneously measuring) amplifiers, each of the 
channels offering an accuracy class of 0.0025 (25 ppm). This is realized by using a 24-bit A/D converter (Sigma-Delta ADC) for each of the amplifier channels.

This new module (Figure 4) should be called Quantum MX 238B belonging to the QuantumX family and referring to the above mentioned famous models with "...38” in their name.

It is based on the principle of carrier frequency ( $\mathrm{CF}$ principle), what has several advantages to be discussed here. The advantages are mainly in the insensitivity to interference and thus in a higher quality of measurement. In the result the use of a carrier frequency allows a much better signal to noise ratio.

HBM, as described in the previous section, also for the reason of good traceability back to the national standard, is using a carrier frequency of $225 \mathrm{~Hz}$ for this new amplifier as well [5].

Hereby the transducer is fed by a sinus-wave voltage and works therefore like a modulator. Details of this can be reviewed in [6], [7], [8].

A further interesting feature is the automatic channel parameterization by TEDS (Transducer Electronic Data Sheet), a technology that allows you to plug in sensors and get started, as a self-recognising process. The characteristics of a transducer are stored inside as an electronic data sheet. The amplifier can import these data from the transducer.

It then converts it automatically into the right settings and gets on with measuring straight away, in the correct units, with no further effort on the customer side. It basically allows you to just plug in and get started and significantly simplifies the handling of sensors. Generally, TEDS can be mounted in the sensor housing or in the plugs of the transducer. When TEDS is mounted in the plug, it should stack with the transducer, preferably with a plug mounting from HBM [10], [11].

For a schematic block diagram of MX 238B, see Figure 6. It is belonging to the HBM QuantumX family, an amplifier family

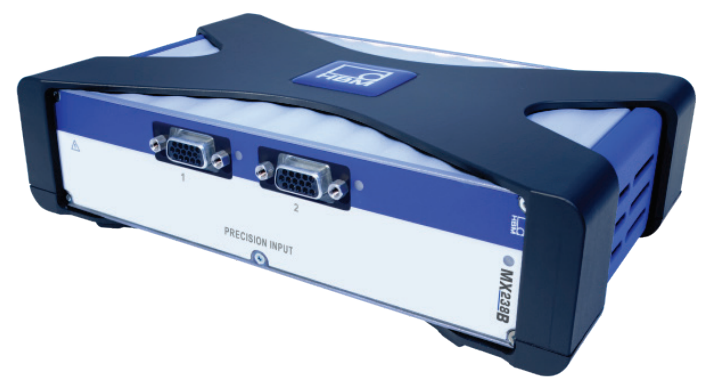

Figure 4. The new amplifier module for any kind of strain gauge transducers named Quantum MX 238B, realized in compact size.

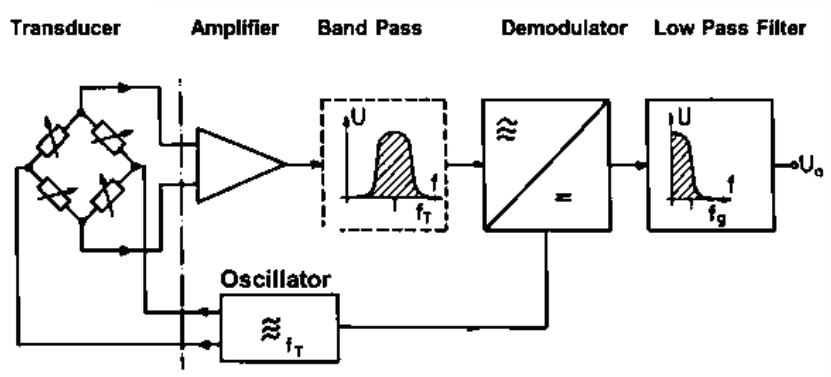

Figure 5. HBM High Precision Amplifiers are based on carrier frequency principle (CF).

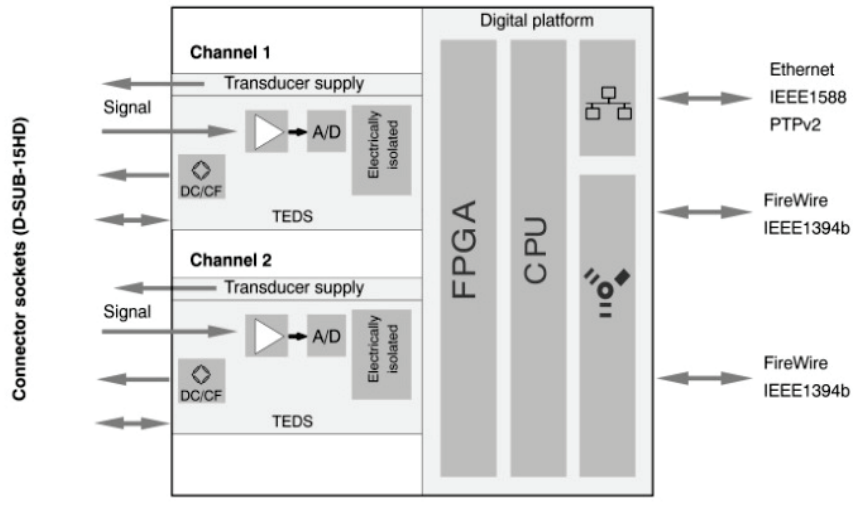

Figure 6. Schematic view of module Quantum MX 238B.

that allows you to process nearly any kind of signal, for instance with the MX840B module which supports as many as 15 different transducer technologies. Module MX410B is also a suitable amplifier for dynamic calibration as it can be seen in [12], [13].

With the introduction of this module QuantumX offers the ability to process and display multiple signals of very different accuracy-bandwidth combinations at the same time. This is one essential advantage of the fact, that MX238B is member of a big family of modules, the HBM main product family QuantumX.

The existing diversity of modules reflects the wide range of measurement tasks, such as needed on test rigs, to perform efficiently with QuantumX by covering many different measurement principles and aspects.

Now the user can choose from a number of modules, namely QuantumX MX238B, QuantumX MX430B, QuantumX MX840B, and QuantumX MX410B in fine steps of very different accuracy-bandwidth combinations. Based on this a real differentiation with respect to the specific measuring task can be done.

All these modules can be connected by IEEE 1394b FireWire and synonymous measurement (in the range of $1 \mu \mathrm{s}$ ) is supported by PTP (precision time protocol) v2.

First time in HBM history we were using a hermetically sealed resistive divider. This component is already very good on its own. However we decided to still implement active temperature compensation.

This additional active temperature compensation is further reducing the already very low temperature coefficient to close up the gap to inductive dividers. This advantage comes with the advantage of miniaturization. Now HBM can offer such a dualchannel module at the same time as compact as this is necessary for a realization in the QuantumX family.

Despite of or even because of using a hermetically sealed resistive divider as a reference, one of the outstanding features of the new amplifier is its independence from temperature for both of the relevant values, so concerning the temperature drift of the zero point and of the span.

For details see Table 1. These results are astonishing, as no other series of precision amplifiers, available on the market, can offer temperature stability in the zero point as well as in the span like this. Note these values refer to the change with 10 Kelvin. In practical laboratory use there should be no temperature dependency at all. By its active temperature compensation Quantum MX238B shows again better than MGCplus ML38B. 
Table 1.Comparison of temperature drift of the different amplifiers.

\begin{tabular}{|c|c|c|c|}
\hline $\begin{array}{l}\text { Precision } \\
\text { Device }\end{array}$ & DMP41 & MGCplusML38B & $\begin{array}{l}\text { Quantum } \\
\text { MX238B }\end{array}$ \\
\hline $\begin{array}{l}\text { Type of voltage } \\
\text { divider }\end{array}$ & \multicolumn{2}{|c|}{ inductive } & resistive \\
\hline $\begin{array}{l}\text { Temperature } \\
\text { drift zero }\end{array}$ & $\begin{array}{c}<2 \mathrm{ppm} / 10 \mathrm{~K} \\
\text { typ. }<1 \mathrm{ppm} / 10 \mathrm{~K}\end{array}$ & $<10 \mathrm{ppm} / 10 \mathrm{~K}$ & $<5 \mathrm{ppm} / 10 \mathrm{~K}$ \\
\hline $\begin{array}{l}\text { Temperature } \\
\text { drift span }\end{array}$ & $\begin{array}{c}<5 \mathrm{ppm} / 10 \mathrm{~K} \\
\text { typ. }<2 \mathrm{ppm} / 10 \mathrm{~K}\end{array}$ & $<20 \mathrm{ppm} / 10 \mathrm{~K}$ & $<10 \mathrm{ppm} / 10 \mathrm{~K}$ \\
\hline
\end{tabular}

The idea of background calibration, first used in DMP41 ([14]-[16]), also has been implemented into the Quantum MX238B. As can be seen in Figure 7, the short-term stability of the Quantum MX238B has been measured. Figure 7 illustrates a measurement plot of a $2 \mathrm{mV} / \mathrm{V}$ signal over 35 hours, where a background-calibration was triggered every minute. During the measurement many such background calibrations are performed. Other user cases have shown that this method has no negative or disturbing influences. The adjustments of the background calibration cycles are in the sub-ppm range. Only the constant thermal noise of the sensor and mainly the voltage noise of the amplifier are recognizable. There is not any interference visible in the noise floor. Thus this demonstrates that it is now possible to calibrate during measurements without any interruption.

Deactivation of the background-calibration does not improve the accuracy, but it will even worsen the accuracy. The user does no longer have to worry about the active control of the previous auto-calibration to get no interruption when measuring.

Possibly we will be able to improve this value again after some time, as we will monitor long term stability the same way, as we have done for a measuring chain consisting out of BN100 and DMP39 for approx. 35 years. Details for this are given in the next section.

\section{FACTORS FOR THE RELIABILITY OF THE MEASURMENT}

In this chapter the following topics, as they are considered important factors of reliability of the measurement, should be discussed. These are:

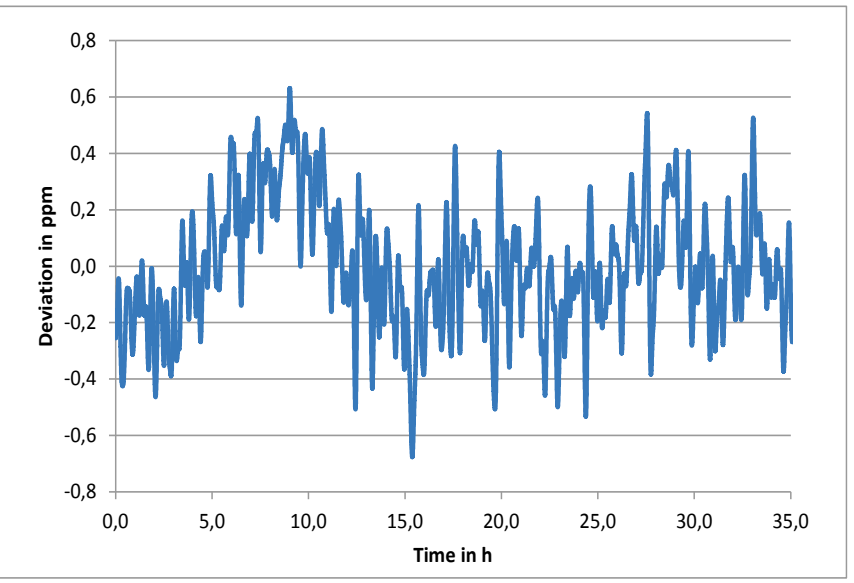

Figure 7. Short-term stability of Quantum MX238B: Measuring with activated Background-Calibration (2.5 mV/V @ 5.0 V rms). a) traceability

b) long term stability

c) referencing the measurement to an international standard

d) ensuring reliable measurement.

First of all traceability is a must. Primary calibration is the highest level, carried out by the National Metrology Institute. Thus metrological applications realize chains of traceability linking measurements made in practice back to reference standards. Such standards are realized by HBM reference transducers and highest precision instruments.

This core concept in metrology - metrological traceability is supported by the outstanding performance of precise products. The main advantages of using strain gauge based technology are, that measurement chains offer an extremely low measurement uncertainty at very good long term stability.

This leads to another factor of measurement reliability. It is the stability and beside of the data of Short-term stability data gathered and described in the previous section, also for longer periods of time the new QuantumX MX238B measuring amplifier shows, as far as this can be evaluated shortly after its introduction, very good results.

As the short term stability is excellent we suppose long-term stability also to be very good, however for this just introduced device, so far, there are no measurements available. First we have specified $<0.0015 \% /$ a with "Auto calibration $>>$ On $<<$ " to be on the safe side and will follow up this over the next years (see Table 2).

Since the introduction of the DMP device series in 1981 we continuously observed the long-term stability of the measuring chain consisting of "DMP39 and BN100"(on the basis of inductances we built our own) and can state that over now more than 35 years its span variation for $+2 \mathrm{mV} / \mathrm{V}$ and -2 $\mathrm{mV} / \mathrm{V}$ signals was every time smaller than the accuracy class of 5 ppm (see following Figure 8) [17].

For electrical calibration of these bridge standards measuring amplifiers are used, which have to be traceable to international standards. As a matter of fact, the standards of HBM and of "Physikalisch-Technische Bundesanstalt", the German National Metrology Institute, are not the same. The reason for this lies in history. HBM needed its own $>>$ Normal $<<(\mathrm{mV} / \mathrm{V})$ to produce first precision amplifiers such as DK37 and DK 38. At that time there was no existing traceability. Then, at the time around 1980, the PTB has set up a normal $\mathrm{mV} / \mathrm{V}$, however these bridge standards showed an offset.

Table 2. Comparison of different amplifiers in terms of their technical data.

\begin{tabular}{|c|c|c|c|}
\hline \multirow{2}{*}{$\begin{array}{l}\text { Comparison } \\
\text { Device Type }\end{array}$} & \multicolumn{3}{|c|}{ Choice of precision instrument } \\
\hline & DMP41 & ML38B & $\mathrm{MX238B}$ \\
\hline $\begin{array}{l}\text { Initial } \\
\text { accuracy }\end{array}$ & $5 \mathrm{ppm}$ & $25 \mathrm{ppm}$ & $25 \mathrm{ppm}$ \\
\hline $\begin{array}{l}\text { Short term } \\
\text { drift }\end{array}$ & $\begin{array}{l}<5 \mathrm{ppm} / 24 \mathrm{~h} \\
\text { typ. }<2 \\
\mathrm{ppm} / 24 \mathrm{~h}\end{array}$ & $<20 \mathrm{ppm} / 24 \mathrm{~h}$ & $<10 \mathrm{ppm} / 24 \mathrm{~h}$ \\
\hline $\begin{array}{l}\text { Long term } \\
\text { stability/a }\end{array}$ & $\begin{array}{l}<5 \mathrm{ppm} / \mathrm{a} \\
\text { typ. }<2 \mathrm{ppm} / \mathrm{a}\end{array}$ & $<25 \mathrm{ppm} / \mathrm{a}$ & $<15$ ppm/a \\
\hline Linearity & $\begin{array}{l}<5 \mathrm{ppm} \\
\text { typ. }<2 \mathrm{ppm}\end{array}$ & $<20 \mathrm{ppm}$ & $<10 \mathrm{ppm}$ \\
\hline $\begin{array}{l}\text { Selectable } \\
\text { Bandwidth } \\
(-3 d B)\end{array}$ & $0.04 \ldots 40 \mathrm{~Hz}$ & $0.03 \ldots 10 \mathrm{~Hz}$ & $0.01 \ldots 50 \mathrm{~Hz}$ \\
\hline
\end{tabular}


Long-Term Stability of the Internal Calibration Method

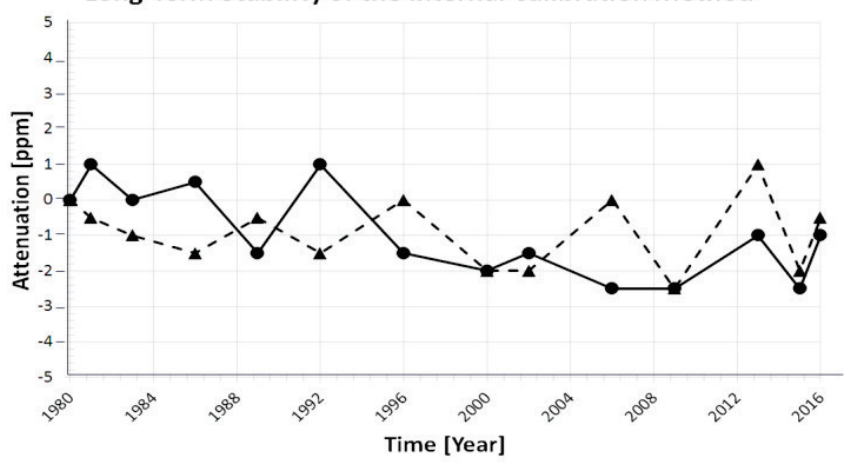

Figure 8. Long term stability of the established calibration method of a measuring chain consisting out of BN100 and DMP39 (+/-).

The standard of PTB is technically constructed differently than the normal in HBM; however, these values can be "translated" into each other. In relation to the values acquired by PTB the end values of all HBM devices with a carrier frequency of $225 \mathrm{~Hz}$, for reasons of continuity, are given 10 ppm lower than the nominal values. There are publications on the details [18], [19].

MX238B demands the connection of the transducers in a six-wire-connection-circuitry (with two additional sensing lines), as for this class of accuracy the resistance of cable lengths in the range of only $\mathrm{cm}$ already counts. Subsequently the device offers also detailed error detection with wire break detection for sensor lines, feeder lines and test leads.

Switchable shunt resistors of $100 \Omega$ allow an artificial bridge detuning. In a $350 \Omega$ Wheatstone bridge, so the standard for the strain gauge pickup principle, there is an upset of 0.886 $\mathrm{mV} / \mathrm{V}$ at $2 \mathrm{mV} / \mathrm{V}$ measuring range, i.e. a fairly significant detuning of approximately $40 \%$.

Regarding a more reliable measurement it can be said that the built-in galvanic isolation ensures avoiding ground loops and therefore the amplifier appears much more robust. Also meeting the high EMC requirements according to EN 61326 for field strengths of $10 \mathrm{~V} / \mathrm{m}$ are a further advantage of the device.

\section{CONCLUSIONS}

In general the market shows a request that the measured signals can be processed more accurately. In this paper it has been shown that there is substantial progress regarding compact size precision amplifiers for strain gauge based transducers such as force, torque and pressure reference transducers used in metrology.

In fact today also amplifiers based on resistive dividers can perform most tasks with sufficient accuracy. For many decades this was not the case. Due to intensive research, HBM can now offer such a dual-channel module at the same time as compact as this is necessary for a realization in the block structure the QuantumX family. It is quite certain, that this previously unknown compactness will open up new fields of integration into applications.

The product family QuantumX now offers the ability to process and display multiple signals of very different accuracybandwidth combinations at the same time.

Thus the decisive progress realized with the QuantumXMX238B is, that now the cooperation with all the other measurement modules of the product family QuantumX with PTPv2 (Precision Time Protocol) timing, allows data processing absolutely simultaneously. Thus the isochronous bus system (Firewire) allows synchronous processing with many other physical quantities.

The precision measurement module MX238B thus combines the advantages of combination with all QuantumXmembers with the possibility of taking measurements with an accuracy class $0.0025(25 \mathrm{ppm})$. This is achieved by the use of the carrier frequency principle and an applied carrier frequency of $225 \mathrm{~Hz}$.

For the first time HBM offers its patented background calibration of this precision class. This ensures that the measurement of the two integrated amplifiers can be continued without interruption during the measurement, so even during the internal calibration.

Furthermore, the transducer can be recognized with the socalled Transducer Electronic Data Sheet ("TEDS") and the individual properties of the transducer can be considered and easily identification of read out.

The measurement results have been showing the very good time as well as temperature stability of resistive dividers.

Nevertheless one has to say that the requirements for a top performance, so amplifiers of highest precision for primary calibration purposes, built to operate on the physical limit (such as our DMP-series), further relies on inductive dividers (voltage ratio derived from the number of windings of coils), as they remain still more accurate than any resistive dividers can be.

\section{REFERENCES}

[1] Hoffmann, K.; An Introduction to Measurementsusing Strain Gauges, Hottinger Baldwin MesstechnikGmbH, Darmstadt, Germany, 1989, pp. 145.

[2] Kreuzer, M.; High-precision measuring technique for strain gauge transducers, internalpublication of Hottinger Baldwin Mestechnik GmbH, Darmstadt, Germany, 1999.

[3] Kleckers, T., Graef, M.; High capacity reference transducer for tensile forces IMEKO 22 $^{\text {nd }}$, TC3, 12th TC5 and 3rd TC22 International Conferences, 2014, Cape Town, South Africa.

[4] Kleckers, T, Precise radial symmetric shear beam force transfer transducer for compression force; XXI IMEKO World Congress, Sept. 2015, Prague, Czech Republic.

[5] Schäfer, A.; Force, strain and pressure transducers based on foil type strain gauges, Proceedings of Eurosensors; XXII conference, Dresden, Germany, October 2008.

[6] Schäfer, A. New possibilities with a high pressure measuring chain consisting of reference pressure transducer P3 TopClassBlueLine and DMP 41 high-precision instrument, PTB Report TH6, Brunswick, Germany, April 2016.

[7] Haller, M. Viel, W.; Schäfer, A.; Enhancement of the measurement characteristics of pressuretransducers up to 15000 bar through monolithicmeasuring design and foil type strain gauges, Proceedings of XIX IMEKO World Congress Lisbon, Portugal, 2009.

[8] Schäfer, A.; et al.; A new type of transducer for accurate and dynamic pressure measurement up to 15000 bar using foil type strain gauges, XVII IMEKO World Congress 2003, Dubrovnik, Croatia, 2003.

[9] Eichstädt. S., Esward, T. J., Schäfer, A. On the necessity of dynamic calibration for improved traceability of mechanical quantities, XXI IMEKO World Congress, Sept. 2015, Prague, Czech Republic.

[10] Schäfer, A.; Dynamic measurements as an emerging field in dynamic metrology; PTBMitteilungen125(2015) Volume 2.

[11] IEEE 1451.4 Standard for Smart Transducers, issued by IEEE 1451.4 Standard Working Group, 1451.2WG, 2004.

[12] Schäfer, A., Challenges in dynamic torque and force measurement with special regard to industrial demands, BIPM 
Workshop on Challenges in Dynamic Measurements.

[13] Schäfer, A., Development results for static and dynamic torque measurement, Proceedings of torque symposium of "ZentrumfürKonstruktionswerkstoffe", MPA (State Material Testing Institute), Darmstadt, Germany, 2014.

[14] Schäck, M., Underestimated impact of measuring cables on high-precision carrier frequency amplifier results and compensation methods therefore, XXI IMEKO World Congress, Sept. 2015, Prague, Czech Republic.

[15] Schäfer, A.; Kitzing, H.; DMP41- a new chapter of ultraprecision instrument for strain gauge transducers, XX IMEKO World Congress, September 2012, Busan, Republic of Korea.

[16] Schäfer, A.; The ultra-precision instrument DMP41-first experience \& appropriate filter settings, IMEKO 22nd TC3,

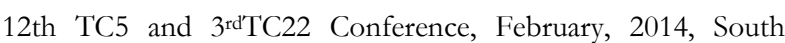
Africa.

[17] Schäck, M.; High-precision measurement of strain gauge transducers at the physical limit without any calibration interruptions; IMEKO 22nd TC3, 12th TC5 and 3rd TC22 International Conferences, February, 2014, South Africa.

[18] Vollmert, R.; Ramm, G.; Realization, maintenance and dissemination of the measurand $>>A C$ voltage ratio in $\mathrm{mV} / \mathrm{V}<<$ for strain gauge measurements, Proceedings of the 17th International Conference of IMEKO TC3, Istanbul, Turkey, 17-21, September, 2001.

[19] Ramm, G. ; Investigation of bridge standards for use in straingauge measurements, Reports in Applied Measurement- RAM, Vol. 6 (1990), No. 1, pp. 26-30. 